\title{
Underutilization of Enhanced Recovery After Surgery (ERAS) in Breast Surgery: An Opportunity to Reduce Opioid Usage
}

\author{
Amy E. Cyr, MD \\ Washington University School of Medicine, Saint Louis, MO
}

Over a 20-year period, opioid-related deaths in the United States have increased sixfold. ${ }^{1}$ In 2017, therefore, the Department of Health and Human Services officially declared this rapid increase in drug-related mortality a public health emergency. During that year, according to the Centers for Disease Control (CDC), more than 70,200 people died from drug overdoses; roughly 47,700 of those deaths involved opioids. Therefore, more people now die of opioid abuse or misuse annually than die of breast cancer. ${ }^{2}$ Although this trend is multifactorial, a sharp increase in the number of opioid prescriptions is considered an important-and addressable-cause. ${ }^{3}$

Despite the opioid crisis being a government and public health priority, the CDC offers little guidance for managing acute postsurgical pain. Although there are CDC pain management guidelines, they are written for primary care providers and make recommendations only for the treatment of chronic pain. ${ }^{4}$ It has, therefore, been up to individual institutions and professional societies to create protocols for controlling surgical pain while addressing the new mandate to prevent opioid dependence.

In this issue, Rao et al. ${ }^{5}$ report the current breast and axillary surgery analgesia practices of members of the American Society of Breast Surgeons (ASBrS), and present the Society's perioperative pain control consensus statement. Roughly $20 \%$ of the 3000 ASBrS members who were invited to take the electronic survey responded. They were queried for methods of pain control used (local anesthesia, blocks, nonsteroidal anti-inflammatory drugs

(C) Society of Surgical Oncology 2020

First Received: 23 December 2019; Published Online: 23 January 2020

A. E. Cyr, MD

e-mail: Amycyr75@gmail.com
[NSAIDs], and opioids) by procedure performed (lumpectomy/excisional biopsy, mastectomy with reconstruction, mastectomy without reconstruction, axillary lymph node dissection [ALND], and oncoplastic rearrangement). Details about the use of each pain control option also were collected.

Local anesthesia for excisional biopsies and lumpectomies was used almost unanimously (98\%) by survey participants, although less frequently for ALNDs (76\%) or mastectomies with or without reconstruction $(49 \%$ and $55 \%$, respectively). Local anesthesia use was not reported for oncoplastic procedures.

Like local anesthesia, NSAIDs were used by nearly all surgeons $(96 \%)$ for excisional biopsies or lumpectomies, but less frequently (75\%) for mastectomies with reconstruction. NSAID use was not reported for mastectomies without reconstruction or for oncoplastic procedures. For the $56 \%$ of respondents who reported using preemptive (immediately preoperative) analgesia, only $57 \%$ used NSAIDs. Surgeons who avoid NSAIDs likely worry about hematoma formation, although the literature does not support this concern. ${ }^{5-7}$

There was significant variability in the use of regional anesthesia for mastectomies with and without reconstruction despite its documented efficacy. ${ }^{8-13}$ Thirty-three percent of surgeons reported never using nerve blocks for patients undergoing mastectomies with reconstruction, and $43 \%$ of surgeons did not use this analgesic strategy at all for patients undergoing mastectomies without reconstruction. The survey, however, did not define "block" and presented only the examples of paravertebral, pectoral nerve, and serratus plane blocks. Epidural anesthesia and intercostal nerve blocks, for instance, also are effective modalities for regional pain control. 
Narcotics were prescribed by approximately $80 \%$ of surgeons after excisional biopsies or lumpectomies and by roughly $90 \%$ of surgeons after ALNDs or mastectomies with or without reconstruction. Although the majority of surgeons $(76 \%)$ reported a reduction in the amount of opioids prescribed, there was marked variability in the number of narcotics prescribed for each procedure. Other investigators have found similar inconsistency in the amounts of prescribed postoperative opioids. ${ }^{3,14}$ In a retrospective study of prescribing practices at their institution, Hill et al. reported a median 20 (range 0-50) opioid pills (a pill was defined as the equivalent of $5 \mathrm{mg}$ of oxycodone) prescribed for patients undergoing partial mastectomy (PM) and a median 20 (range 0-60) pills for those undergoing PM with sentinel lymph node biopsy (SLNB). They calculated that opioid requirements could be met for $80 \%$ of their patients by prescribing five pills following PM and ten pills following PM with SLNB. ${ }^{3}$

In response to a paucity of guidance for postoperative narcotic prescribing, a panel consisting of multiple stakeholders, including surgeons, pain management providers, pharmacists, and patients published a single-institution consensus for quantities of opioids to prescribe for common surgical procedures. PM with and without SLNB were 2 of the 20 general surgery procedures for which the panel made recommendations regarding the range of tablets (defined as $5 \mathrm{mg}$ of oxycodone per tablet) to prescribe: $0-15$ with and $0-10$ without SLNB. The panel unanimously agreed that all patients should be offered nonnarcotic analgesia and concluded that not prescribing narcotics was a reasonable option for all of these procedures, based on individual factors, including patient preference. ${ }^{15}$

Rao et al. make similar recommendations for how many narcotic pain pills to prescribe, depending on the procedure, although the authors do not define the morphine milligram equivalent of a tablet. The only procedures for which the workgroup recommends prescribing more than 10 tablets are ALND (20 or fewer tablets), mastectomy with or without axillary surgery (20 or fewer), and mastectomy with reconstruction ( 30 or fewer). ${ }^{5}$

In addition to making these prescribing recommendations, the ASBrS workgroup encourages the use of regional anesthesia and strongly recommends the use of Enhanced Recovery After Surgery (ERAS) programs. Only a minority (14\%) of respondents in this study reported using ERAS protocols. ${ }^{5}$ This is a clear opportunity for improving breast surgical care and reducing narcotic use. The authors do not, however, define ERAS or its components, nor do they provide data on whether ERAS use is increasing among the respondents.
The ERAS $^{\circledR}$ Society, conceived in 2001, publishes guidelines for multiple specialties, including general, thoracic, orthopedic, urologic, reconstructive, and gynecologic surgery. ${ }^{16}$ ERAS provides an evidence-based framework for perioperative care to reduce morbidity and mortality, and it is quickly becoming the standard of care for surgical patients, both in the United States and abroad. ${ }^{17-21}$ A key ERAS recommendation is the use of multimodal analgesia, including preemptive analgesia and regional anesthesia, to reduce the use of perioperative opioids, which contribute to ileus, delayed mobilization, and postoperative nausea and vomiting. Although not intended to prevent opioid dependence, ERAS protocols should help by minimizing the need for narcotics for postoperative pain control. ${ }^{22}$

In summary, ERAS is an effective, evidence-based tool widely embraced by other surgical subspecialties and with the potential to reduce opioid requirements of breast surgery patients. I congratulate the ASBrS for addressing the opioid crisis, providing these recommendations, and encouraging the adoption of ERAS pathways. This consensus statement should spark interest in nonopioid analgesia options and educate breast surgeons on the need for ERAS. The next step is for the breast surgery community to define more clearly the pillars of ERAS as they apply to our patients and, ultimately, to measure its impact on patient outcomes, including perioperative opioid needs.

DISCLOSURES The author has no conflict of interest to disclose.

\section{REFERENCES}

1. CDC Opioid Basics. https://www.cdc.gov/drugoverdose/epidemi c/index.html. Accessed 22 Dec 2019.

2. U.S. Breast Cancer Statistics. https://www.breastcancer.org/symp toms/understand_bc/statistics. Accessed 22 Dec 2019.

3. Hill MV, McMahon ML, Stucke RS, Barth RJ. Wide variation and excessive dosage of opioid prescriptions for common general surgical procedures. Ann Surg. 2017;265(4):709-14.

4. Dowell D, Haegerich TM, Chou R. CDC guideline for prescribing opioids for chronic pain-United States, 2016. MMWR Recomm Rep. 2016;65(No. RR-1):1-49. https://doi.org/10.15585/ mmwr.rr6501e1.

5. Rao R, Jackson RS, Rosen B, et al. Pain control in breast surgery: survey of current practice and recommendations for optimizing management American Society of Breast Surgeons opioid/pain control workgroup. Ann Surg Oncol. 2020. https://doi.org/10.12 45/s10434-020-08197-z

6. Sharma S, Chang DW, Koutz C, et al. Incidence of hematoma associated with ketorolac after TRAM flap breast reconstruction. Plast Reconstr Surg. 2001;107:352-5.

7. Gobble RM, Hoang HL, Kachniarz B, Orgill DP. Ketorolac does not increase perioperative bleeding: a meta-analysis of randomized controlled trials. Plast Reconstr Surg. 2014;133:741-55.

8. Cheng GS, Ilfeld BM. An evidence-based review of the efficacy of perioperative analgesic techniques for breast cancer-related surgery. Pain Med. 2017;18(7):1344-65.

9. Temple-Oberle C, Shea-Budgell MA, Mark T, et al. Consensus review of optimal perioperative care in breast reconstruction: 
enhanced recovery after surgery (ERAS) society recommendations. Plast Reconstr Surg. 2017;139(5):1056e-71e.

10. Bashandy GM, Abbas DN. Pectoral nerves I and II blocks in multimodal analgesia for breast cancer surgery: a randomized clinical trial. Reg Anesth Pain Med. 2015;40(1):68-74.

11. Humble SR, Dalton AJ, Li L. A systematic review of therapeutic interventions to reduce acute and chronic post-surgical pain after amputation, thoracotomy, or mastectomy. Eur J Pain. 2015;19(4):451-65.

12. Beverly A, Kaye AD, Ljungqvist O, Urman RD. Essential elements of multimodal analgesia in enhanced recovery after surgery (ERAS) guidelines. Anesthesiol Clin. 2017;35(2):e115-43

13. Offodile AC, Gu C, Boukovalas S, Coroneos CJ, Chatterjee A, Largo RD, Butler C. Enhanced recovery after surgery (ERAS) pathways in breast reconstruction: systematic review and metaanalysis of the literature. Breast Cancer Res Treat. 2019;173(1):65-77.

14. Mooromid MJ, Blay E, Holl JL, Bilimoria KY, Johnson JK, Eskandari MK, Stulberg JJ. Discharge prescription patterns of opioid and nonopioid analgesics after common surgical procedures. Pain Rep. 2018;3(1):e637.

15. Overton HN, Hanna MN, Bruhn WE, Hutfless S, Bicket MC, Makary MA, Opioids After Surgery Workgroup. Opioid-prescribing guidelines for common surgical procedures: an expert panel consensus. J Am Coll Surg. 2018;227(4):411-418.

16. ERAS ${ }^{\circledR}$ Society List of Guidelines. https://erassociety.org/guide lines/list-of-guidelines/. Accessed 23 Dec 2019
17. Fearon KC, Ljungqvist O, Von Meyenfeldt M, et al. Enhanced recovery after surgery: a consensus review of clinical care for patients undergoing colonic resection. Clin Nutr. 2005;24(3):466-77. Epub 2005 Apr 21. https://doi.org/10.1016/ j.clnu.2005.02.002.

18. Joliat GR, Ljungqvist O, Wasylak T, Peters, Demartines N. Beyond surgery: clinical and economic impact of Enhanced Recovery After Surgery programs. BMC Health Serv Res. 2018;18:1008. https://doi.org/10.1186/s12913-018-3824-0.

19. Brown JK, Singh K, Dumitru R, Chan E, Kim MP. The benefits of enhanced recovery after surgery programs and their application in cardiothoracic surgery. Methodist Debakey Cardiovasc J. 2018;14(2):77-88.

20. Jones MR, Viswanath O, Peck J, Kaye AD, Jatinder SG, Simopoulos TT. A brief history of the opioid epidemic and strategies for pain medicine. Pain Ther. 2018;7(1):13-21.

21. Arsalani-Zadeh R, El Fadl D, Yassin N, MacFie J. Evidencebased review of enhancing postoperative recovery after breast surgery. Br J Surg. 2011;98: 181-196.

22. Rojas KE, Manasseh DM, Flom PL, Agbroko S, Bilbro N, Andaz C, Borgen PI. A pilot study of a breast surgery Enhanced Recovery After Surgery (ERAS) protocol to eliminate narcotic prescription at discharge. Breast Cancer Res Treat. 2018;171(3):621-6.

Publisher's Note Springer Nature remains neutral with regard to jurisdictional claims in published maps and institutional affiliations. 